\title{
Clinical efficacy of conjunctival flap surgery in the treatment of refractory fungal keratitis
}

\author{
HONORINE NIZEYIMANA ${ }^{1}$, DAN-DAN ZHOU ${ }^{2}$, XIU-FEN LIU ${ }^{1}$, XIAO-TAO PAN ${ }^{1}$, CONG LIU $^{1}$, \\ CHENG-WEI LU ${ }^{1}$ and JI-LONG HAO ${ }^{1}$
}

Departments of ${ }^{1}$ Ophthalmology and ${ }^{2}$ Radiology, The First Hospital of Jilin University, Changchun, Jilin 130021, P.R. China

Received July 24, 2016; Accepted April 10, 2017

DOI: 10.3892/etm.2017.4605

\begin{abstract}
The aim of the present study was to investigate the use and effectiveness of a selective, partial, pedunculated (tongue-shaped) conjunctival flap (CF) for the treatment of refractory fungal keratitis (FK) with or without perforation. A total of 31 cases of corneal diseases treated by CF surgery between April 2014 and October 2015 were evaluated. Among the 31 cases, 16 cases (male:female, 11:5) with FK were selected. Logistic regression analysis was used to investigate factors associated with complications of CF surgery. A higher prevalence of FK was identified among male farmers compared with female farmers, in which plant trauma was the most prevalent cause of the disease. Only 4 patients had experienced corneal perforation prior to $\mathrm{CF}$ surgery. Patients aged 61-80 years had a higher prevalence of FK (50\%) compared with other age groups; however, there was no statistically significant correlation between the prevalence of FK and sex or age. It was also demonstrated that age, sex, combined surgery and surgery duration were not significantly associated with post-surgical complications. All CF surgeries were performed following corneal ulcer scraping; however, 4 patients $(12.5 \%)$ required additional surgery. The visual acuity of participants post-surgery decreased in 4 cases and remained unchanged in 12 cases. A total of 3 study patients experienced post-surgical complications of corneal perforation (1 patient) and purulent exudate spreading (2 patients). The post-surgical outcome was good for all study participants as the surgeries were able to control infection and preserve the eyeball, with the potential of future corneal transplant. These results suggest that $\mathrm{CF}$ surgery may be a useful alternative treatment for refractory
\end{abstract}

Correspondence to: Dr Cheng-Wei Lu or Dr Ji-Long Hao, Department of Ophthalmology, The First Hospital of Jilin University, 71 Xinmin Street, Changchun, Jilin 130021, P.R. China

E-mail: 1cwchina800@sina.com

E-mail: 289736582@qq.com

Key words: conjunctival flap, fungal, keratitis, refractory, surgery treatment
FK in countries such as China where there is lack of cornea donors.

\section{Introduction}

Fungal keratitis (FK) was first described by Leber (1) in 1879 as a suppurative, ulcerative and sight-threatening infection of the cornea caused by fungi. Significant damage to the eye, such as blindness, may occur if FK is not diagnosed early or left untreated for long periods of time. Compared with bacterial keratitis, FK has a poor prognosis, and the condition accounts for almost $50 \%$ of all cases of infectious keratitis in developing countries $(2,3)$. Currently available topical antifungal drugs have limitations, such as limited spectra of activity and surface toxicity (4-6). The clinical efficacy of antifungal drugs depends on the concentration achieved in ocular tissue, which, in turn, depends on the molecular mass, route of administration, duration of contact, and ability of the compound to penetrate the eye (7). Some antifungal agents have a high molecular mass exceeding 500 Da (such as amphotericin B, natamycin and ketoconazole), leading to their poor penetration, even if they are lipophilic in nature (6). FK responds poorly to antifungal agents, and thus surgical intervention in the form of therapeutic keratoplasty is required more frequently in cases of FK compared with bacterial keratitis $(3-6,8)$. However, the shortage of corneal donors for keratoplasty in countries such as China has made conjunctival flap (CF) surgery a promising alternative for the treatment of refractory FK. This method is an alternative to the Gunderson's flaps technique that involves covering the whole cornea (9), which has the disadvantage of making it difficult to observe the anterior chamber or monitor disease progression.

In the present study, the efficacy of a tongue-shaped partial flap was evaluated in study subjects. Specifically, the study assessed the use of selective, partial, pedunculated (tongue-shaped) CF for the treatment of refractory FK with or without perforation, and investigated the effectiveness of this method in managing FK.

\section{Materials and methods}

Ethical approval. The present study was approved by the Ethics Committee of the First Hospital of Jilin University 
Table I. Patient clinical characteristics and outcomes.

\begin{tabular}{|c|c|c|c|c|c|c|c|c|c|c|}
\hline $\begin{array}{l}\text { Patient } \\
\text { number }\end{array}$ & $\begin{array}{c}\text { Age } \\
\text { (years) }\end{array}$ & Sex & Diagnosis & $\begin{array}{c}\text { Surgical } \\
\text { duration } \\
\text { (min) }\end{array}$ & $\begin{array}{c}\text { Pre- } \\
\text { operative } \\
\text { VA }\end{array}$ & $\begin{array}{c}\text { Post- } \\
\text { operative } \\
\text { VA }\end{array}$ & $\begin{array}{l}\text { Further } \\
\text { surgery }\end{array}$ & $\begin{array}{c}\text { Post- } \\
\text { operative } \\
\text { hospitalization } \\
\text { (days) }\end{array}$ & Occupation & Complications \\
\hline 1 & 81 & $\mathrm{M}$ & FK & 30 & 0.4 & 0.4 & CAD & 3 & Farmer & $\mathrm{N}$ \\
\hline 2 & 68 & $\mathrm{~F}$ & FK & 30 & $\mathrm{CF} / 30 \mathrm{~cm}$ & HM & CAD & 3 & Farmer & $Y^{a}$ \\
\hline 3 & 86 & $\mathrm{~F}$ & FK & 60 & NPL & NPL & $\mathrm{CAD}+\mathrm{ACL}$ & 3 & Other & $\mathrm{N}$ \\
\hline 4 & 65 & $\mathrm{M}$ & $\mathrm{FK}+\mathrm{P}$ & 30 & LP & LP & CAD & 6 & Farmer & $\mathrm{N}$ \\
\hline 5 & 77 & $\mathrm{M}$ & $\mathrm{FK}+\mathrm{P}$ & 40 & HM & HM & CAD & 5 & Farmer & $\mathrm{N}$ \\
\hline 6 & 78 & $\mathrm{~F}$ & FK & 60 & LP & LP & $\begin{array}{l}\text { CAD+ } \\
\text { ACL+ER }\end{array}$ & 18 & Farmer & $\mathrm{Y}^{\mathrm{b}}$ \\
\hline 7 & 58 & M & FK & 60 & 0.1 & 0.1 & CAD & 7 & Farmer & $\mathrm{Y}^{\mathrm{b}}$ \\
\hline 8 & 62 & $\mathrm{M}$ & FK & 60 & 0.12 & 0.12 & CAD & 9 & Farmer & $\mathrm{N}$ \\
\hline 9 & 47 & $\mathrm{M}$ & FK & 85 & 0.01 & $\mathrm{CF} / 20 \mathrm{~cm}$ & CAD & 4 & Farmer & $\mathrm{N}$ \\
\hline 10 & 52 & M & FK & 60 & $\mathrm{CF} / 20 \mathrm{~cm}$ & $\mathrm{CF} / 20 \mathrm{~cm}$ & CAD & 17 & Other & $\mathrm{N}$ \\
\hline 11 & 63 & M & FK & 30 & $\mathrm{HM} / 50 \mathrm{~cm}$ & $\mathrm{HM} / 50 \mathrm{~cm}$ & CAD & 7 & Farmer & $\mathrm{N}$ \\
\hline 12 & 60 & $\mathrm{M}$ & $\mathrm{FK}+\mathrm{P}$ & 40 & $\mathrm{HM} / 30 \mathrm{~cm}$ & $\mathrm{HM} / 30 \mathrm{~cm}$ & $\mathrm{CAD}$ & 3 & Farmer & $\mathrm{N}$ \\
\hline 13 & 39 & M & FK & 30 & $\mathrm{CF} / 30 \mathrm{~cm}$ & $\mathrm{HM} / 20 \mathrm{~cm}$ & $\mathrm{CAD}$ & 4 & Farmer & $\mathrm{N}$ \\
\hline 14 & 74 & M & FK & 30 & $\mathrm{HM} / 30 \mathrm{~cm}$ & $\mathrm{HM}$ & $\mathrm{CAD}$ & 9 & Farmer & $\mathrm{N}$ \\
\hline 15 & 70 & $\mathrm{~F}$ & FK & 80 & 0.3 & 0.3 & CAD & 3 & Farmer & $\mathrm{N}$ \\
\hline 16 & 58 & $\mathrm{~F}$ & $\mathrm{FK}+\mathrm{P}$ & 30 & $\mathrm{HM} / 10 \mathrm{~cm}$ & LP & CAD & 4 & Farmer & $\mathrm{N}$ \\
\hline
\end{tabular}

${ }^{a}$ Corneal perforation. ${ }^{b}$ Purulent exudate spreading. NPL, no perception of light; LP, perception of light; HM, hand movements; CF, counting fingers; CAD, corneal ulcer debridement; ACL, anterior chamber lavage; P, perforation; ER, eyeball removal.

(Changchun, China), and all study patients provided written informed consent prior to their participation in the study.

Study design and patients. The present study was a non-comparative retrospective study. Study subjects were recruited from the Department of Ophthalmology at the First Hospital of Jilin University. A total of 31 patients who had undergone CF surgery for treatment of corneal diseases between April 2014 and October 2015 at the hospital were assessed. Of these, 16 patients who were diagnosed with FK were selected for further investigation, according to the criteria below. Study participants were divided into four age groups: <40, 40-60, 61-80, and >80 years old. Patient clinical characteristics are listed in Table I.

Inclusion and exclusion criteria of the patients. The inclusion criteria were as follows: i) Patients from the Department of Ophthalmology; ii) Patients of all ages; iii) admitted patients; and iv) patients with FK. Participants were excluded based on the following criteria: i) Patients from ophthalmologic hospitals; ii) outpatients; and iii) patients with other types of keratitis.

Diagnosis and surgery type. The diagnosis of FK was confirmed based on in vivo confocal microscopy findings. In all study patients, surgery was performed using a selective, partial, pedunculated (tongue-shaped) flap to cover only the ulcerated part of the cornea, as opposed to covering the whole cornea.
Surgical procedure. Surgery was performed in a sterile surgical room with the patient lying in supine position under an operating microscope. Retrobulbar anesthesia was administered with $3 \mathrm{ml} \mathrm{2 \%}$ lidocaine (Shanghai Fuxing Chaohui Pharmaceutical Co., Ltd., Shanghai, China) to the diseased eye and 5\% povidone iodine (Jilin Ytai Mingxing Medicine Co., Ltd.) was applied with a cotton ball to sterilize the skin around it. The diseased eye was draped and an eye spectrum was inserted. The surgeon used surgical blades to remove the corneal epithelium and any necrotic tissue from the ulcerated cornea or within $0.5-1 \mathrm{~mm}$ of the ulcer margin. The surgeon selected a conjunctival segment close to the ulcer that exhibited the best blood supply to the corneal ulcer. A $1 \mathrm{ml}$ subconjunctival injection of balanced 2\% lidocaine (Shanghai Fuxing Chaohui Pharmaceutical Co., Ltd.) with 1:100,000 epinephrine (Suicheng Pharmaceutical Co., Ltd., Xinzheng, China) was administered to help separate the conjunctiva from the underlying Tenon's capsule. The injected solution was spread over the inflated conjunctiva using a cotton-tipped applicator to stop the conjunctiva from stretching. Blunt-tipped scissors and blunt forceps were used to hold the conjunctiva while the surgical blade was used to make a tongue-shaped incision in the quadrant of the conjunctiva near the ulcerated region. Only a partial segment of the conjunctiva was mobilized on the ulcerated part of the cornea After setting the conjunctival segment in place, a 10-0 monofilament nylon suture was used to secure the flap in the cornea using interrupted sutures. Finally, one drop of $0.5 \%$ Tropicamide Phenylephrine Eye Drops (Santen Pharmaceutical Co., Ltd., Osaka, Japan) and 

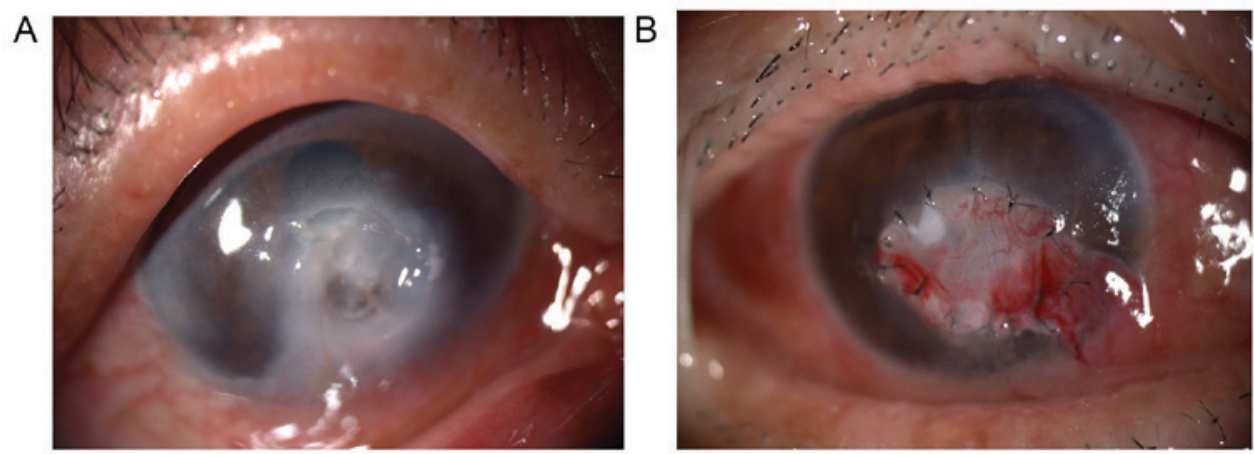

Figure 1. Slit lamp examination of a patient with refractory fungal keratitis (A) prior to and (B) after conjunctival flap surgery. A deep $5 \times 5 \mathrm{~mm}$ ulcer was located at the center of the cornea and hypopion was also present. At 1 week post-surgery, a conjunctival flap was covering the corneal ulcer and the hypopion was no longer visible.

Table II. Sex, age, and age-sex distributions of patients.

\begin{tabular}{lccc}
\hline Variable & Value & t-test $/ \chi^{2}$-value & P-value \\
\hline Mean age & & 14.50 & 0.141 \\
$\quad$ Male $(\mathrm{n}=11)$ & 61.64 & & \\
Female (n=5) & 72.00 & & \\
Sex & & 2.25 & 0.134 \\
Male & 11 & & \\
Female & 5 & & \\
Age groups (years) & & $7.50^{\mathrm{e}}$ & 0.058 \\
$\quad<40$ & 1 & & \\
$40-60$ & 5 & & \\
$61-80$ & 8 & & \\
$>80$ & 2 & & \\
\hline
\end{tabular}

$\sim 0.3 \mathrm{~g}$ of antibiotic Ofloxacin eye ointment $(3.5 \mathrm{~g}: 10.5 \mathrm{mg}$; Santen Pharmaceutical Co., Ltd.) were introduced into the eye, and the eye was dressed. The dressing was changed every $24 \mathrm{~h}$ and, depending on the condition of the patient, sutures were removed from 2 weeks after surgery. Fig. 1 depicts an ulcerated cornea in one patient before and after CF surgery.

Statistical analysis. Both descriptive and inferential statistical analyses were performed. For categorical variables, the descriptive analyses included both numbers and percentages. $\chi^{2}$ and Mann-Whitney tests were used to investigate differences in the distribution of study patients based on age and sex. Using surgical complications as dependent variables and age, sex, combined surgery and surgical duration as independent variables, simple and multiple logistic regression analyses (inferential statistics) were used to investigate factors associated with the occurrence of complications after CF surgery. SPSS 18.0 software (SPSS, Inc., Chicago, IL, USA) was used for all statistical analyses, and $\mathrm{P}<0.05$ was considered to indicate a statistically significant difference.

\section{Results}

Patient characteristics. A total of 16 patients were diagnosed with FK and underwent CF surgery. Among these 16 patients,
$11(68.8 \%)$ were male and 5 (31.2\%) were female; however, there was no statistical difference in the gender ratio of patients (Table I). Male farmers comprised the majority of the patient cohort $(n=11,68.8 \%)$, and plant trauma was the primary cause of disease. The $<40,40-60,61-80$ and $>80$ years age groups had 1, 5, 8 and 2 cases, respectively. The 61-80 years old group had the largest number of patients (50.0\%). The results of the present study demonstrated that the sex, age group and age-sex distributions of study patients did not differ significantly (Table II).

Patient outcomes. Among the study patients, 4 presented with perforation associated with FK prior to surgery. Corneal scraping was performed on all patients prior to CF surgery. Among the 16 patients enrolled, 14 patients underwent CF combined with corneal ulcer debridement (without anterior chamber lavage), while 2 patients underwent anterior chamber lavage in addition to CF surgery and corneal ulcer debridement. A total of 3 patients experienced post-operative complications; 1 patient exhibited corneal perforation, 1 patient ultimately had his eyeball surgically removed 10 days after CF due to the uncontrolled spreading of purulent exudates (hypopion) beyond the anterior chamber; and another patient with hypopion in the anterior chamber underwent an anterior chamber lavage plus further CF surgery and healed well. The remaining 13 patients recovered well. Postoperative visual acuity (VA) was decreased in 4 cases (25\%) and remained unchanged in 12 cases $(75 \%)$. The post-surgical outcome was good for all patients that underwent surgeries with controlled eye infections and preserved eyeballs. Simple and multiple logistic regression analyses indicated that age, sex, surgery duration, and combined surgeries were not significantly associated with the development of complications (Table III).

\section{Discussion}

Due to different fungal pathogens, complex clinical manifestations and limited antifungal medications, FK is typically difficult to treat, which leads to worsening of the condition and the need for surgical treatment (10). This has resulted in higher rates of flap surgeries as a non-pharmacological treatment for FK (11). Surgical intervention for patients with refractory FK 
Table III. Logistic regression analyses of factors influencing conjunctival flap post-surgery complications.

\begin{tabular}{lccc}
\hline Variable & B & OR $(95 \%$ CI $)$ & P-value \\
\hline Simple logistic regression & & & \\
Age & -0.03 & $0.97(0.88-1.08)$ & 0.63 \\
Sex & -1.90 & $0.15(0.01-2.29)$ & 0.17 \\
Surgical duration & -0.01 & $0.99(0.93-1.06)$ & 0.77 \\
Combined surgery & -1.81 & $0.16(0.01-2.55)$ & 0.20 \\
Multiple logistic regression & & & \\
Age & 0.24 & $1.27(0.85-1.91)$ & 0.24 \\
Sex & -3.54 & $0.03(0.00-9.23)$ & 0.23 \\
Surgical duration & 0.07 & $1.07(0.93-1.23)$ & 0.33 \\
Combined surgery & -5.54 & $0.00(0.00-17.68)$ & 0.20
\end{tabular}

OR, odds ratio; CI, confidence interval.

is effective, and CF surgery is a simple and economical option that typically preserves the eye and vision.

The present study included more males $(68.8 \%)$ than females $(31.2 \%)$, though this difference was not statistically significant. Khater et al (12) performed an epidemiological study of 66,303 patients (264 cases of mycotic keratitis), and in comparing mycotic keratitis with nonmycotic keratitis, it was demonstrated that both mycotic and nonmycotic keratitis were markedly more prevalent in males compared with females. Contrary to results of the present study, a number of studies have reported a significant correlation between sex and the distribution of FK patients, whereby males were more affected by FK than females (13-16). These studies, including that by Khater et al (12), reported a higher prevalence of FK among male farmers compared with female farmers, and that plant trauma was the primary cause of the disease. The results of the present study were in accordance with this, with FK being most prevalent in farmers.

Contrary to our results, which suggested that FK was more prevalent in the 61-80 years age group, previous studies have reported that FK was more prevalent in young adults $(8,15)$. However, some studies $(15,17)$ demonstrated a prevalence in males between 50-60 years of age. Khater et al (12) also observed that mycotic keratitis was most prevalent in the $40-60$ years age group (52.4\% of cases) (12). The results of the present study differ from those of most of other authors, possibly due to the relatively small sample used in the present study.

In the majority of patients enrolled in the present study, the aim of CF surgery was to prevent or stop corneal perforation and/or inflammation and to preserve the eyeball, rather than to improve the patient's vision. As such, the decreased or unchanged post-operative VA observed in the present study does not indicate that CF surgery was unsuccessful. In a previous study investigating the different outcomes of Gundersen flap in which sutures were administered in one group and glue in another, Chung and Mehta (18) reported unchanged post operative VA in 5/7 patients, and increased VA in 2/7 patients, with all 7 patients diagnosed with different types of corneal disorders (18). In the present study, the tongue-shaped flap was mobilized only on the ulcerated cornea, as opposed to covering the whole cornea. This method provides an alternative to Gunderson's flaps, and avoids the complications associated with the Gunderson's flaps technique (9). If the conjunctiva covers the entire cornea, it obstructs any view of the anterior chamber $(19,20)$, which increases the difficulty of monitoring disease progression and checking intraocular pressure to avoid glaucoma development. Furthermore, patients with short fornices have been subjected to the Gunderson's flaps technique, where it was observed that the technique may lead to the development of ptosis (19). These limitations favored alternative approaches to treatment (21-26).

The majority of patients in the present study underwent CF surgery combined with corneal ulcer debridement $(\mathrm{CF}+\mathrm{CAD})$; however, 2 patients underwent $\mathrm{CF}$ surgery with corneal ulcer debridement and anterior chamber lavage (CF $+\mathrm{CAD}+\mathrm{ACL}$ ). Zeng et al (27) investigated the effectiveness of an amniotic membrane covering on the ulcerated cornea following debridement in a patient with FK, and concluded that corneal scraping prior to surgical therapy (in this case amniotic membrane) promotes healing of the cornea. McGrath and Lee demonstrated in 2015 (28) that corneal epithelial debridement may be used as an effective therapy for ocular surface diseases as well as diagnosis. Among 62 eyes that received corneal epithelial debridement for diagnostic purposes, 48 were positive for infective keratitis, among which 5 eyes (20.8\%) were diagnosed with FK. Corneal scraping is an effective treatment for pathologies of the corneal surface $(29,30)$.

In the present study, 3 patients experienced post-surgical complications; 1 patient exhibited corneal perforation, another exhibited uncontrolled spreading of purulent exudates (hypopion) beyond the anterior chamber, and 1 patient exhibited hypopion in the anterior chamber. The patient with cornea perforation received a cornea transplant shortly after CF surgery. The patient with uncontrolled hypopion spreading beyond the anterior chamber underwent an anterior chamber lavage, though ultimately had their eyeball surgically removed 10 days after CF. The patient with hypopion in the anterior chamber underwent an anterior chamber lavage plus further CF surgery, which ultimately succeeded in controlling the infection. Based on Table III, age, sex, surgery duration and combined surgeries were not statistically associated with the probability of developing post-surgical complications. However, it was noted that the majority of complications were associated with higher disease severity, due to its propensity to spread and/or failure to heal after surgery. Many patients seek medical care at an advanced stage of the disease when treatment at local clinics has failed. In the present study, no patients developed any of the most common post-surgical complications associated with CF surgery, such as conjunctival buttonholes and flap retractions. This suggests that subsequent complications did not result from a failure in the CF surgery itself, but may be associated with the severity of the disease. However, the relatively small sample used in the present study may have skewed data; therefore, future studies with larger sample sizes are now required to verify the current results.

Although keratoplasty is among the best treatment strategies for refractory FK with or without perforation, CF surgery may be a useful alternative treatment in cases where immediate corneal transplant is not feasible. In the present study, 
the outcome was good in all the patients with the exception of 1 patient, who ultimately required surgical removal of the eyeball. The results of the present study suggest that $\mathrm{CF}$ is an effective treatment for refractory FK when corneal transplantation is not a possibility.

\section{Acknowledgements}

The present study was supported by the Development and Reform Commission of Jilin Province (grant no. 2015Y031-1) and the First Hospital of Jilin University (grant no. JDYY72016055).

\section{References}

1. Leber TH: Keratomycosis spergillina als ursache von hypopyonkeratites. Graefes Ach Clin Exp Ophthalmol 25: 285-301, 1879.

2. Ou JI and Acharya NR: Epidemiology and treatment of fungal corneal ulcers. Int Ophthalmol Clin 47: 7-16, 2007.

3. Gopinathan U, Sharma S, Garg P and Rao GN: Review of epidemiological features, microbiological diagnosis and treatment outcome of microbial keratitis: Experience of over a decade. Indian J Ophthalmol 57: 273-279, 2009.

4. Johns KJ and O'Day DM: Pharmacologic management of keratomycoses. Surv Ophthalmol 33: 178-188, 1988.

5. O'Day DM, Head WS, Robinson RD and Clanton JA: Corneal penetration of topical amphotericin B and natamycin. Curr Eye Res 5: 877-882, 1986

6. Kaur IP, Rana C and Singh H: Development of effective ocular preparations of antifungal agents. J Ocul Pharmacol Ther 24 481-493, 2008.

7. Kaur IP and Kakkar S: Topical delivery of antifungal agents. Expert Opin Drug Deliv 7: 1303-1327, 2010.

8. Sony P, Sharma N, Vajpayee RB and Ray M: Therapeutic keratoplasty for infectious keratitis: A review of the literature. CLAO J 28: 111-118, 2002.

9. Sandinha T, Zaher SS, Roberts F, Devlin HC, Dhillon B and Ramaesh K: Superior forniceal conjunctival advancement pedicles (SFCAP) in the management of acute and impending corneal perforations. Eye (Lond) 20: 84-89, 2006.

10. Dursun D, Fernandez V, Miller D and Alfonso EC: Advanced fusarium keratitis progressing to endophthalmitis. Cornea 22: 300-303, 2003.

11. Sanitato JJ, Kelley CG and Kaufman HE: Surgical management of peripheral fungal keratitis (keratomycosis). Arch Ophthalmol 102: 1506-1509, 1984.

12. Khater MM, Shehab NS and El-Badry AS: Comparison of mycotic keratitis with nonmycotic keratitis: An epidemiological study. J Ophthalmol 2014: 254302, 2014.
13. Rautaraya B, Sharma S, Kar S, Das S and Sahu SK: Diagnosis and treatment outcome of mycotic keratitis at a tertiary eye care center in eastern India. BMC Ophthalmol 11: 39, 2011.

14. Bharathi MJ, Ramakrishnan R, Vasu S, Meenakshi R and Palaniappan R: Epidemiological characteristics and laboratory diagnosis of fungal keratitis. A three-year study. Indian J Ophthalmol 51: 315-321, 2003.

15. Chander J and Sharma A: Prevalence of fungal corneal ulcers in northern India. Infection 22: 207-209, 1994.

16. Deshpande SD and Koppikar GV: A study of mycotic keratitis in Mumbai. Indian J Pathol Microbiol 42: 81-87, 1999.

17. Saha S, Banerjee D, Khetan A and Sengupta J: Epidemiological profile of fungal keratitis in urban population of West Bengal India. Oman J Ophthalmol 2: 114-118, 2009.

18. Chung HW and Mehta JS: Fibrin glue for Gundersen flap surgery. Clin Ophthalmol 7: 479-484, 2013.

19. Gundersen T: Conjunctival flaps in the treatment of corneal disease with reference to a new technique of application. AMA Arch Ophthalmol 60: 880-888, 1958.

20. Lin DT, Webster RG Jr and Abbott RL: Repair of corneal lacerations and perforations. Int Ophthalmol Clin 28: 69-75, 1988.

21. Saini JS, Sharma A and Grewal SP: Chronic corneal perforations. Ophthalmic Surg 23: 399-402, 1992.

22. Hirst LW, Smiddy WE and Stark WJ: Corneal perforations. Changing methods of treatment, 1960-1980. Ophthalmology 89: 630-635, 1982.

23. Portnoy SL, Insler MS and Kaufman HE: Surgical management of corneal ulceration and perforation. Surv Ophthalmol 34: 47-58, 1989

24. Weiss JL, Williams P, Lindstrom RL and Doughman DJ: The use of tissue adhesive in corneal perforations. Ophthalmology 90: 610-615, 1983.

25. Hirst LW and De Juan E Jr: Sodium hyaluronate and tissue adhesive in treating corneal perforations. Ophthalmology 89: 1250-1253, 1982

26. Sharma A, Kaur R, Kumar S, Gupta P, Pandav S, Patnaik B and Gupta A: Fibrin glue versus N-butyl-2-cyanoacrylate in corneal perforations. Ophthalmology 110: 291-298, 2003.

27. Zeng B, Wang P, Xu LJ, Li XY, Zhang H and Li GG: Amniotic membrane covering promotes healing of cornea epithelium and improves visual acuity after debridement for fungal keratitis. Int J Ophthalmol 7: 785-789, 2014.

28. McGrath LA and Lee GA: Corneal epithelial debridement for diagnosis and therapy of ocular surface disease. Clin Exp Optom 98: 155-159, 2015.

29. Kenyon KR: Review of surgical strategies for ocular surface disease. Ocul Surf 9: 164-168, 2011.

30. Jeng BH, Dupps WJ Jr, Meisler DM and Schoenfield L: Epithelial debridement for the treatment of epithelial basement membrane abnormalities coincident with endothelial disorders. Cornea 27: 1207-1211, 2008. 